
\title{
3 Research Square \\ Co-Cultivation of Sake Yeast and Kocuria Isolates From the Sake Brewing Process
}

\section{Momoka Terasaki}

Toyama Prefectural University

\section{Airu Inoue}

Toyama Prefectural University

\section{Saki Yoshida}

Toyama Prefectural University

\section{Masato Yamada}

Narimasa Sake Brewery

\section{Hiroshi Toda}

Toyama Prefectural University

Hiromi Nishida ( $\square$ hnishida@pu-toyama.ac.jp )

Graduate School of Engineering and Department of Biotechnology, Toyama Prefectural University, 5180 Kurokawa, Imizu, Toyama 939-0398, Japan

\section{Research Article}

Keywords: Kocuria, Narimasa Sake Brewery, TGY media, koji solution

Posted Date: January 20th, 2021

DOI: https://doi.org/10.21203/rs.3.rs-148211/v1

License: (c) (i) This work is licensed under a Creative Commons Attribution 4.0 International License.

Read Full License 


\section{Abstract}

Kocuria isolates collected from the sake brewing process have inhabited the Narimasa Sake Brewery in Toyama, Japan. To investigate the effect of these actinobacterial isolates on the growth and metabolism of sake yeast, co-cultivation of sake yeast and Kocuria isolates was performed in a medium containing tryptone, glucose, and yeast extract (TGY), and a solution containing koji (steamed rice covered with Aspergillus oryzae) and glucose. In the TGY medium, the ethanol concentration and the number of living cells of each microorganism were measured. In the koji solution, the concentrations of ethanol and organic acids (citric acid, lactic acid, and succinic acid) were measured. The results showed that in TGY media, the growth of each Kocuria isolate in the co-culture of the two Kocuria isolates was similar to that in each monoculture. However, the growth of both Kocuria isolates was inhibited in the co-cultures of sake yeast and Kocuria isolates. On the other hand, the growth and ethanol productivity of sake yeast did not differ between its monoculture and co-cultures with Kocuria isolates. In the koji solution, although the concentration of succinic acid did not differ between the monocultures of sake yeast and co-cultures with Kocuria isolates, the concentrations of citric acid and lactic acid were different, indicating that Kocuria isolates affected the microbial population and/or sake yeast metabolism, but the effects were not identical between the two isolates. This strongly suggests that bacteria inhabiting a sake brewery influence the flavor of sake products of the brewery.

\section{Introduction}

During sake brewing, the koji mold Aspergillus oryzae converts rice starch into glucose, which is then converted into ethanol by the sake yeast Saccharomyces cerevisiae. Eventually, the ethanol concentration reaches approximately $20 \%$. Most microorganisms present at the beginning of the sake production process die because of the high ethanol concentration, although some lactic acid bacteria can grow at high ethanol concentrations and cause spoiling ${ }^{1}$. Thus, sake undergoes pasteurization during storage, a process which was recorded 300 years before Pasteur reported his method.

Living bacteria cannot be detected in sake products, but bacterial DNA can be detected. Based on DNA sequence data, we obtained information on bacteria that have entered and briefly grown during sake brewing $^{2-5}$. Living bacteria were previously isolated at the beginning of the sake production process ${ }^{6}$. Among these, bacteria belonging to the genus Kocuria were the most common isolates found in the Narimasa Sake Brewery (Brewery Toyama 1 in Terasaki and Nishida, 20206). Kocuria belongs to the phylum Actinobacteria ${ }^{7}$. It has been reported that lactic acid bacteria belonging to the phylum Firmicutes play a role in the sake production process ${ }^{1-3,8}$. In addition, co-cultivation of sake yeast and lactic acid bacteria has also been performed ${ }^{9,10}$. However, to our knowledge, no bacteria belonging to Actinobacteria have been reported to be associated with sake brewing.

In this study, a medium containing tryptone, glucose, and yeast extract (TGY), a solution containing koji (steamed rice covered with Aspergillus oryzae), and glucose were used for cultivation (Fig. 1). Although the TGY medium was autoclaved and kept sterile, koji included koji mold and other microorganisms. Koji 
is commercially available in Japan. Although sake yeasts produce ethanol in both the TGY medium and koji solution, environments in koji solution are more similar to those in sake brewing than those in the TGY medium.

Kocuria DNA was detected only in the products of Narimasa Sake Brewery, suggesting that the Kocuria isolates have inhabited the sake brewery. In addition, Kocuria DNA was detected not in clear sake, but in cloudy sake and sake-kasu (sake lee) ${ }^{6}$. This indicates that Kocuria cells do not lyse in sake even after they die because these cells are difficult to lyse using ethanol ${ }^{11}$. Kocuria cells are then squeezed via filtration and included in the sake-kasu fraction during the process of sake brewing.

The composition of amino acids and organic acids is associated with bacterial flora in the sake $\mathrm{e}^{12,13}$. Some bacteria are alive and grow temporarily during the sake production process, but finally die in the final sake product. The Kocuria strains may have inhabited the Narimasa Sake Brewery, which may further play a key role in the interaction among microorganisms, and influence the flavor and taste of sake. If so, we could produce sakes with different flavors and tastes by exchanging the sake breweryspecific bacteria from different sake breweries for the sake production process. Taken together, the purpose of this study was to elucidate whether Kocuria isolate(s) can affect the growth and metabolism of sake yeast during co-cultivation.

\section{Results And Discussion}

\section{Monoculture and co-culture of Kocuria isolates in TGY medium}

In this study, the Kocuria isolates TGY1120_3 and TGY1127_2 were used, which were identified based on different colony colors (Fig. 2). Comparison of monocultures of the isolates TGY1120_3 and TGY1127_2 at different temperatures showed that these two Kocuria isolates grew better at $15^{\circ} \mathrm{C}$ than at $5{ }^{\circ} \mathrm{C}$ or 30 ${ }^{\circ} \mathrm{C}$ (Fig. 3A). Comparison of the 16S rDNA sequence showed that the isolates TGY1120_3 (accession number: NZ_AP022830) and TGY1127_2 (NZ_AP022834) belonged to K. koreensis and K. uropygioeca, respectively. The optimal growth temperature of $K$. koreensis was between $30^{\circ} \mathrm{C}$ and $37^{\circ} \mathrm{C}^{14}$, while that of $K$. uropygioeca was $35^{\circ} \mathrm{C}^{15}$. Considering that sake brewing was performed at $15^{\circ} \mathrm{C}$, the isolates TGY1120_3 and TGY1127_2 probably adapted to grow at $15^{\circ} \mathrm{C}$. If this is true, these Kocuria isolates have inhabited and evolved in the Narimasa Sake Brewery for a long time.

The growth of either isolate TGY1120_3 or TGY1127_2 in the co-culture of these two isolates was similar to that in each monoculture (Fig. 3A). The maximum growth rate of isolate TGY1127_2 was higher than that of the isolate TGY1120_3 (Fig. 3A). To elucidate this, a cluster analysis ${ }^{6}$ of the eight growth patterns at $15^{\circ} \mathrm{C}$ and $30^{\circ} \mathrm{C}$ was performed using the software $\mathrm{R}$ (http://www.R-project.org/). The results indicated that the isolates TGY1120_3 and TGY1127_2 were clustered separately, except for TGY1127_2, during coculture at $30^{\circ} \mathrm{C}$ (Fig. 3B).

\section{Co-culture between sake yeast and Kocuria isolates in TGYm2 medium}


Sake yeast grew in co-cultures with Kocuria isolate(s) as well as in monoculture (Fig. 4). In addition, the change in ethanol concentration was similar among monocultures and in three different co-cultures (Fig. 4). In both co-cultures of isolate TGY1120_3 and sake yeast and that of isolate TGY1127_2 and sake yeast, the Kocuria isolates did not grow but they survived (Fig. 4). The isolates TGY1120_3 and TGY1127_2 survived up to 19 and 25 days, respectively (Fig. 4).

On the other hand, in the co-culture containing sake yeast and two Kocuria isolates, the Kocuria isolates grew from 0 to 5 days (Fig. 4) and the number of living cells was limited to $10^{5}-10^{6}$ cells $/ \mathrm{mL}$ after five days (Fig. 4). During the same period, the number of living cells decreased (Fig. 4). In the co-culture containing sake yeast and both Kocuria isolates, the isolates TGY1120_3 and TGY1127_2 survived up to 14 and 25 days, respectively (Fig. 4). Interestingly, although the ethanol concentration was above $5 \%$ after eight days in the monoculture and co-cultures of sake yeast and either Kocuria isolate, it was below $5 \%$ after eight days in the co-cultures containing sake yeast and both Kocuria isolates. Considering that the growth of sake yeast did not differ among the four different culture conditions (Fig. 4), the ethanol productivity of sake yeast may decrease after eight days in the co-cultures of sake yeast and the two Kocuria isolates. The low ethanol productivity of sake yeast may be associated with the growth of Kocuria isolates from 0 to 5 days of co-cultivation.

Kocuria DNA was not detected in clear sakes, but was detected in cloudy sakes and sake-kasu ${ }^{6}$, because Kocuria cells are difficult to lyse using ethanol ${ }^{11}$. In this study, TGYm2 medium used after 34 days of incubation in a co-culture of sake yeast and both Kocuria isolates was centrifuged to separate it into a precipitate and supernatant. Quantitative PCR (qPCR) was performed on each of them using primers specific to genomic DNA of the isolates TGY1120_3 and TGY1127_2. Living cells were not detected after 34 days of incubation. The qPCR results showed that Kocuria DNA was detected in the precipitate but not in the supernatant, supporting the idea that Kocuria cells are difficult to lyse using ethanol. In addition, the Cq value of TGY1120_3-specific qPCR was higher than that of the TGY1127_2-specific qPCR, with a difference of approximately 3 (Table 1), indicating that the number of dead or non-culturable ${ }^{16}$ cells in cultures containing the isolate TGY1127_2 was eight times greater than that in the cultures containing the isolate TGY1120_3. This indicates that the Kocuria cells of isolates TGY1120_3 and TGY1127_2 are difficult to lyse after co-cultivation.

\section{Co-culture of sake yeast and Kocuria isolates in koji solution}

The change in ethanol concentration during co-cultivation of sake yeast and the Kocuria isolate TGY1120_3 was similar to that during monocultivation of sake yeast (Fig. 5). On the other hand, ethanol productivity in the co-culture of sake yeast and isolate TGY1127_2 was slightly higher than that in the monoculture of sake yeast (Fig. 5). During co-culture with sake yeast, ethanol concentration in the coculture with both Kocuria isolates was higher than those in the other cultures (Fig. 5). The cause of this ethanol productivity in the co-culture of sake yeast and both Kocuria isolates is uncertain. It is possible that the existence of Kocuria isolates became a stress for sake yeast, and hence the yeast produced more ethanol. 
The change in citric acid concentration was different between the monoculture of sake yeast and the cocultures containing yeast and Kocuria isolates (Fig. 5). However, the concentration of citric acid was similar for the first five days of culture between the monoculture of sake yeast and co-cultures of yeast and Kocuria isolate(s) (Fig. 5), and the citric acid concentration in the co-culture of sake yeast and isolate TGY1120_3 was higher than that in the monoculture of sake yeast at 17 and 23 days. On the other hand, citric acid concentrations in the co-cultures containing sake yeast and isolate TGY1127_2 and in the cocultures containing sake yeast and both Kocuria isolates were higher at 17 days but lower at 23 days than in the monoculture of sake yeast (Fig. 5).

The lactic acid concentration increased in the monoculture of sake yeast (Fig. 5). Due to the growth of lactic acid bacteria, lactic acid concentration increases during the production of sake fermentation starters kimoto ${ }^{2}$ and yamahai-moto ${ }^{3}$. In the koji solution, lactic acid concentration peaked after 10 days and then decreased after 17 days in the co-cultures of yeast and Kocuria isolates (Fig. 5). This may indicate that the koji solution, containing lactic acid bacteria and Kocuria isolates, may affect the growth and/or metabolism of lactic acid bacteria.

The change in succinic acid concentration was similar among the four culture conditions. It decreased after 5 days of culture, then increased up to 23 days (Fig. 5). This indicates that the TCA cycle changed from oxidative metabolism to reductive metabolism in the sake yeast, starting from five days in culture ${ }^{17}$.

The amino acid compositions of the final cultures of koji solution were similar among the four culture conditions (Fig. 6). It indicates that Kocuria isolates do not affect the amino acid metabolism in sake yeast.

\section{Conclusions}

Kocuria isolates did not affect the growth of sake yeast during co-cultivation. However, the composition and concentration of organic acids were influenced by the presence of Kocuria isolate(s) in the koji solution. These results strongly suggest that the addition or temporary growth of Kocuria isolate(s) during sake brewing could alter the flavor and taste of sake. Thus, bacteria that have entered and briefly grown during sake brewing may have had effects on sake flavor and taste when they were grown with sake yeast.

\section{Methods}

\section{Strains used in this study}

Sake yeast (Kyokai yeast strain 1401) ${ }^{18}$ and the Kocuria isolates ${ }^{6}$ TGY1120_3 and TGY1127_2 were used in this study.

\section{Media used in this study}


For the cultivation of Kocuria isolates, we used the following media: TGY (tryptone $5 \mathrm{~g} / \mathrm{L}$, glucose $1 \mathrm{~g} / \mathrm{L}$, yeast extract $3 \mathrm{~g} / \mathrm{L}$ ), TGYm1 (tryptone $20 \mathrm{~g} / \mathrm{L}$, glucose $20 \mathrm{~g} / \mathrm{L}$, yeast extract $10 \mathrm{~g} / \mathrm{L}$ ), TGYm2 (tryptone 20 $\mathrm{g} / \mathrm{L}$, glucose $300 \mathrm{~g} / \mathrm{L}$, yeast extract $10 \mathrm{~g} / \mathrm{L}$ ), TGY agar (yeast extract $3 \mathrm{~g} / \mathrm{L}$, tryptone $5 \mathrm{~g} / \mathrm{L}$, glucose $1 \mathrm{~g} / \mathrm{L}$, agar $15 \mathrm{~g} / \mathrm{L}$, cycloheximide $500 \mathrm{ng} / \mathrm{mL}$ ), and TGYm1c agar (yeast extract $10 \mathrm{~g} / \mathrm{L}$, tryptone $20 \mathrm{~g} / \mathrm{L}$, glucose $20 \mathrm{~g} / \mathrm{L}$, agar $15 \mathrm{~g} / \mathrm{L}$, cycloheximide $500 \mathrm{ng} / \mathrm{mL}$ ).

For the cultivation of sake yeast (Saccharomyces cerevisiae strain 1401), we used the following media: TGYm1 and TGYm1k agar (yeast extract $10 \mathrm{~g} / \mathrm{L}$, tryptone $20 \mathrm{~g} / \mathrm{L}$, glucose $20 \mathrm{~g} / \mathrm{L}$, agar $15 \mathrm{~g} / \mathrm{L}$, kanamycin $500 \mathrm{ng} / \mathrm{mL}$ ).

To cultivate a mixture of Kocuria isolate(s) and sake yeast, we used the medium TGYm2.

\section{Monocuture of Kocuria isolates}

The Kocuria isolate TGY1120_3 or TGY1127_2 was statically cultured in TGY medium at $5{ }^{\circ} \mathrm{C}, 15^{\circ} \mathrm{C}$, or $30{ }^{\circ} \mathrm{C}$. Sampling was performed at $0,5,8,14,19,25$, and 33 days of incubation. Each sample was diluted $1: 10$, and $5 \mu \mathrm{L}$ of each sample was spread on TGY agar to count the number of colonies.

\section{Co-culture of Kocuria isolates}

The Kocuria isolates TGY1120_3 and TGY1127_2 were statically cultured in TGY medium at $15^{\circ} \mathrm{C}$ or 30 ${ }^{\circ} \mathrm{C}$. Sampling was performed at 0, 5, 8, 14, 19, 25, and 33 days of incubation. Each sample was diluted 10 times, and $5 \mu \mathrm{L}$ of each sample was spread on TGY agar to count the number of colonies. The colony colors of the isolates TGY1120_3 and TGY1127_2 were cream and orange, respectively (Fig. 2). Thus, the number of colonies could be counted according to the presence of different colors.

\section{Co-culture of sake yeast and either Kocuria isolates}

Kocuria isolate TGY1120_3 and sake yeast strain 1401, or isolate TGY1127_2 and yeast strain 1401 were statically cultured together in TGYm2 medium at $15^{\circ} \mathrm{C}$. Sampling was performed at $0,5,8,14,19,25$, and 33 days of incubation. Each sample was diluted 10 times, and $5 \mu \mathrm{L}$ of each sample was spread on TGYm1c agar to count the number of Kocuria isolate colonies, and on TGYm1k agar to count the number of sake yeast colonies. In addition, the ethanol concentration of each broth at each sampling time was determined using HPLC.

Furthermore, koji solution ( $k o j i 100 \mathrm{~g} / \mathrm{L}$, glucose $200 \mathrm{~g} / \mathrm{L}$ ) was used in the co-culture of sake yeast and Kocuria isolates. Koji from Shin-mura Koujimiso in Toyama, Japan, was used in this study, and the resulting mixture of koji, glucose, and water was called the koji solution. Co-cultures containing sake yeast and TGY1120_3, and sake yeast and TGY1127_2 were statically grown in koji solution at $15^{\circ} \mathrm{C}$. Sampling was performed at $0,5,10,17$, and 23 days of incubation. The concentrations of ethanol and organic acids in each sample were detected using HPLC.

\section{Co-culture of sake yeast and both Kocuria isolates}


The Kocuria isolates TGY1120_3 and TGY1127_2 and the sake yeast strain 1401 were statically cultured together in TGYm2 medium at $15^{\circ} \mathrm{C}$. Sampling was performed at 0, 5, 8, 14, 19, 25, and 33 days of incubation. Each sample was diluted 10 times, and $5 \mu \mathrm{L}$ of each sample was spread on TGYm1c agar to count the number of colonies of Kocuria isolates, and on TGYm1k agar to count the number of sake yeast colonies. In addition, the ethanol concentration of each broth at each sampling time was determined using HPLC.

In addition, co-cultures containing sake yeast and both Kocuria isolates (TGY1120_3 and TGY1127_2) were statically performed in koji solution at $15^{\circ} \mathrm{C}$. Sampling was performed at $0,5,10,17$, and 23 days of incubation. The concentrations of ethanol and organic acids in each sample were detected using HPLC.

\section{Quantitative PCR (qPCR) for DNA-specific detection of Kocuria isolates}

One milliliter of TGYm2 medium, at 34 days of incubation, containing a co-culture of sake yeast and both Kocuria isolates was centrifuged to separate it into a precipitate and supernatant. The precipitate was then resuspended in $100 \mu \mathrm{L}$ of water. The TGY1120_3 DNA-specific primers used in this study were 5'CTGGAGATCGGTGGGTTCAC-3' and 5'-TGAGAATCGCGAACCCCTTC-3'. The TGY1127_2 DNA-specific primers used in this study were 5'-CGACAAGACTCACCTGTTCG-3' and 5'-CTCGACGTCGTAGCAGATCA-3'. Approximately $2.5 \mu \mathrm{L}$ of each sample was used for qPCR, which was performed using a LightCycler Nano (Roche, Basel). The cycling conditions were as follows: $95^{\circ} \mathrm{C}$ for $10 \mathrm{~min}$, followed by 45 cycles of denaturation $\left(95^{\circ} \mathrm{C}\right.$ for $\left.10 \mathrm{~s}\right)$, annealing and extension $\left(70^{\circ} \mathrm{C}\right.$ for $\left.30 \mathrm{~s}\right)$. After the final extension, a melting curve cycle was performed from $60^{\circ} \mathrm{C}$ to $95^{\circ} \mathrm{C}$, at increments of $0.1^{\circ} \mathrm{C} / \mathrm{s}$, to confirm the absence of nonspecific PCR products. The quantification cycle $(\mathrm{Cq})$ values were obtained using the LightCycler Nano Software (Roche, Basel, Switzerland).

\section{Quantification of ethanol and organic acids using HPLC}

High-performance liquid chromatography (HPLC) analysis of the TGY medium and koji solution during cultivation was carried out using a Shimadzu LC-10 HPLC system equipped with an Aminex HPX-87H column (7.8 mm × 300 mm; Rio-Rad, Tokyo). Five microliters of each sample solution was injected and isocratically eluted with $5 \mathrm{mM} \mathrm{H}_{2} \mathrm{SO}_{4}$ at a flow rate of $0.6 \mathrm{~mL} / \mathrm{min}$. The column temperature was maintained at $35^{\circ} \mathrm{C}$, and the compounds were monitored with a UV detector at $210 \mathrm{~nm}$ and a Shodex RI71 refractive index detector (Showa Denko, Tokyo). The retention time of each compound was as follows: 9.6 min for citric acid, 12.4 min for succinic acid, 13.4 min for lactic acid, and 22.0 min for ethanol.

\section{Quantification of amino acids using HPLC}

The amino acid compositions of the final cultures of koji solution were assessed via HPLC by IDEA Consultants, Inc. (https://ideacon.jp/). One $\mathrm{mL}$ of each sample and $3 \mathrm{~mL}$ of $80 \%$ methanol were mixed with vigorous stirring. After that, the solution was sonicated for 5 min and then centrifuged at 3,000 rpm for $10 \mathrm{~min}$. The supernatant was filtered using $0.2 \mu \mathrm{m}$ pore size filter. Labeling was performed by autoinjector program (IDEA Consultants). 


\section{Declarations}

\section{Acknowledgments}

This work was supported by research funds of Toyama Prefecture (to H.N.) and JSPS KAKENHI Grant Number 20J14864 (to M.T.).

\section{Author contributions}

M.T., A.I., S.Y., M.Y., and H.T. carried out the experiments. H.N. designed the experiments. M.T., H.T., and H.N. wrote the paper.

\section{Competing interests}

The authors declare no competing interests.

\section{References}

1. Suzuki, K., Asano, S., lijima, K. \& Kitamoto, K. Sake and beer spoilage lactic acid bacteria-a review. J. Inst. Brew.114, 209-223 (2008).

2. Bokulich, N.A., Ohta, M., Lee, M. \& Mills, D.A. Indigenous bacteria and fungi drive traditional kimoto sake fermentations. Appl. Environ. Microbiol.80, 5522-5529 (2014).

3. Koyanagi, T. et al. Tracing microbiota changes in yamahai-moto, the traditional Japanese sake starter. Biosci. Biotechnol. Biochem.80, 399-406 (2016).

4. Terasaki, M., Fukuyama, A., Takahashi, Y., Yamada, M. \& Nishida, H. Bacterial DNA detected in Japanese rice wines and the fermentation starters. Curr. Microbiol.74, 1432-1437 (2017).

5. Terasaki, M., Miyagawa, S., Yamada, M. \& Nishida, H. Detection of bacterial DNA during the process of sake production using sokujo-moto. Curr. Microbiol.75, 874-879 (2018).

6. Terasaki, M. \& Nishida, H. Bacterial DNA diversity among clear and cloudy sakes, and sake-kasu. Open Bioinf. J.13, 74-82 (2020).

7. Stackebrandt, E., Koch, C., Gvozdiak, O. \& Schumann, P. Taxonomic dissection of the genus Micrococcus: Kocuria gen. nov., Nesterenkonia gen. nov., Kytococcus gen. nov., Dermacoccus gen. nov., and Micrococcus Cohn 1872 gen. emend. Int. J. Syst. Evol. Microbiol.45, 682-692 (1995).

8. Taniguchi, M. et al. Growth-inhibitor of hiochi bacteria in namazake (raw sake) by bacteriocins from lactic acid bacteria. J. Biosci. Bioeng.109, 570-575 (2010).

9. Kawarai, T., Furukawa, S., Ogihara, H. \& Yamasaki, M. Mixed-species biofilm formation by lactic acid bacteria and rice wine yeasts. Appl. Environ. Microbiol.73, 4673-4676 (2007).

10. Watanabe, D. et al. Metabolic switching of sake yeast by kimoto lactic acid bacteria through the $\left[G A R^{+}\right]$non-genetic element. J. Biosci. Bioeng.126, 624-629 (2018). 
11. Fujita, K. et al. The cell structural properties of Kocuria rhizophila for aliphatic alcohol exposure. Enzyme Microb. Technol.39, 511-518 (2006).

12. Akaike, M. et al. Chemical and bacterial components in sake and sake production process. Curr. Microbiol.77, 632-637 (2020).

13. Tsuji, A., Kozawa, M., Tokuda, K., Enomoto, T. \& Koyanagi, T. Robust domination of Lactobacillus sakei in microbiota during traditional Japanese sake starter yamahai-moto fermentation and the accompanying changes in metabolites. Curr. Microbiol.75, 1498-1505 (2018).

14. Park, E.-J. et al. Kucuria koreensis sp. nov., isolated from fermented seafood. Int. J. Syst. Evol. Microbiol.60, 140-143 (2010).

15. Braun, M.S., Wang, E., Zimmermann, S., Boutin, S. \& Wink, M. Kocuria uropygioeca sp. nov. and Kocuria uropygialis sp. nov., isolated from the preen glands of Great Spotted Woodpeckers (Dendrocopos major). Syst. Appl. Microbiol.41, 38-43 (2018).

16. Colwell, R.R. \& Grimes, D.J. Nonculturable microorganisms in the environment. ASM Press, Herndon, USA (2000).

17. Muratsubaki, $\mathrm{H}$. Regulation of reductive production of succinate under anaerobic conditions in baker's yeast. J. Biochem.102, 705-714 (1987).

18. Ohya, Y. \& Kashima, M. History, lineage and phenotypic differentiation of sake yeast. Biosci. Biotechnol. Biochem.83, 1442-1448 (2019).

\section{Tables}

Table 1. Results of quantitative PCR. 


\begin{tabular}{|lll|}
\hline Sample & Primers & Cq value \\
\hline Supernatant & TGY1127_2 & Not detected \\
\hline Supernatant & TGY1127_2 & Not detected \\
\hline Supernatant & TGY1127_2 & Not detected \\
\hline Precipitate & TGY1127_2 & 19.8 \\
\hline Precipitate & TGY1127_2 & 20.8 \\
\hline Precipitate & TGY1127_2 & 21.4 \\
\hline Supernatant & TGY1120_3 & Not detected \\
\hline Supernatant & TGY1120_3 & Not detected \\
\hline Supernatant & TGY1120_3 & Not detected \\
\hline Precipitate & TGY1120_3 & 17.7 \\
\hline Precipitate & TGY1120_3 & 17.7 \\
\hline Precipitate & TGY1120_3 & 17.6 \\
\hline
\end{tabular}

Figures 


\section{Cultures using TGYm2 medium}

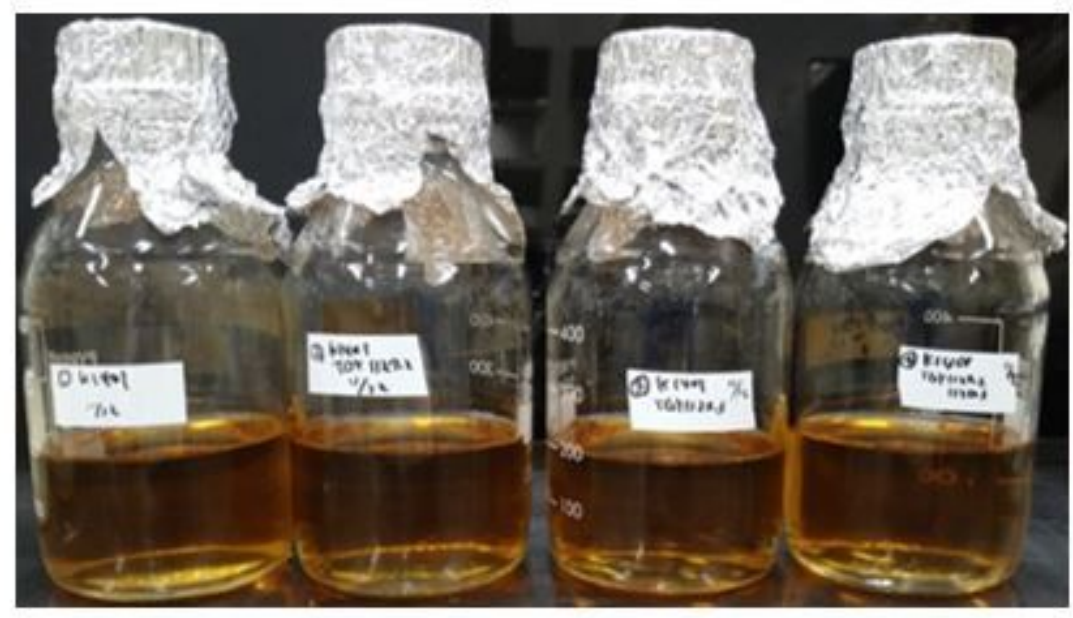

\section{Cultures using koji solution}

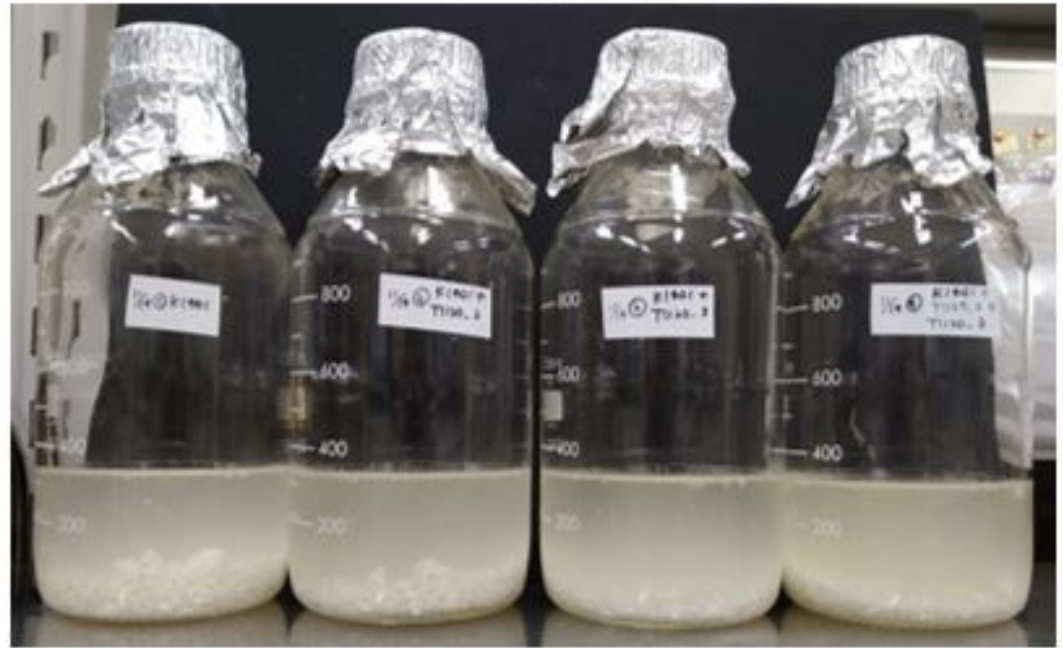

Figure 1

Cultivation of sake yeast and Kocuria isolate(s) in TGYm2 medium and koji solution. TGYm2 medium contained tryptone $20 \mathrm{~g} / \mathrm{L}$, glucose $300 \mathrm{~g} / \mathrm{L}$, and yeast extract $10 \mathrm{~g} / \mathrm{L}$. Koji solution contained koji 100 $\mathrm{g} / \mathrm{L}$ and glucose $200 \mathrm{~g} / \mathrm{L}$. 


\section{Isolate TGY1127_2}

\section{Isolate TGY1120_3}

\section{$5 \mu \mathrm{m}$}

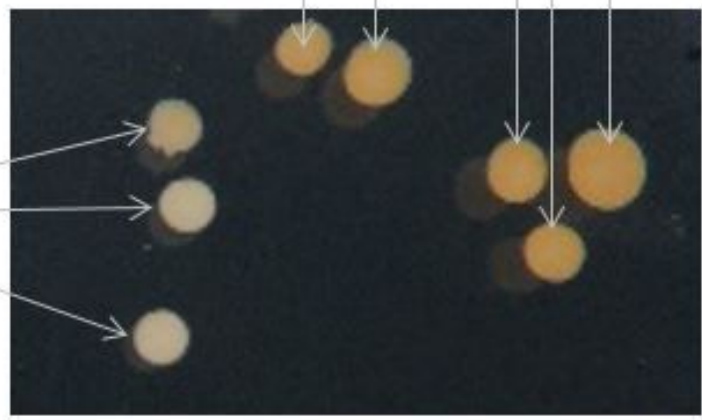

\section{Kocuria colonies on TGY agar}

Figure 2

Colonies of Kocuria isolates grown on TGY agar. The isolates TGY1120_3 and TGY1127_2 appeared as cream and orange colonies, respectively. Phase contrast microscopy images were captured using an Olympus BX51 microscope. 

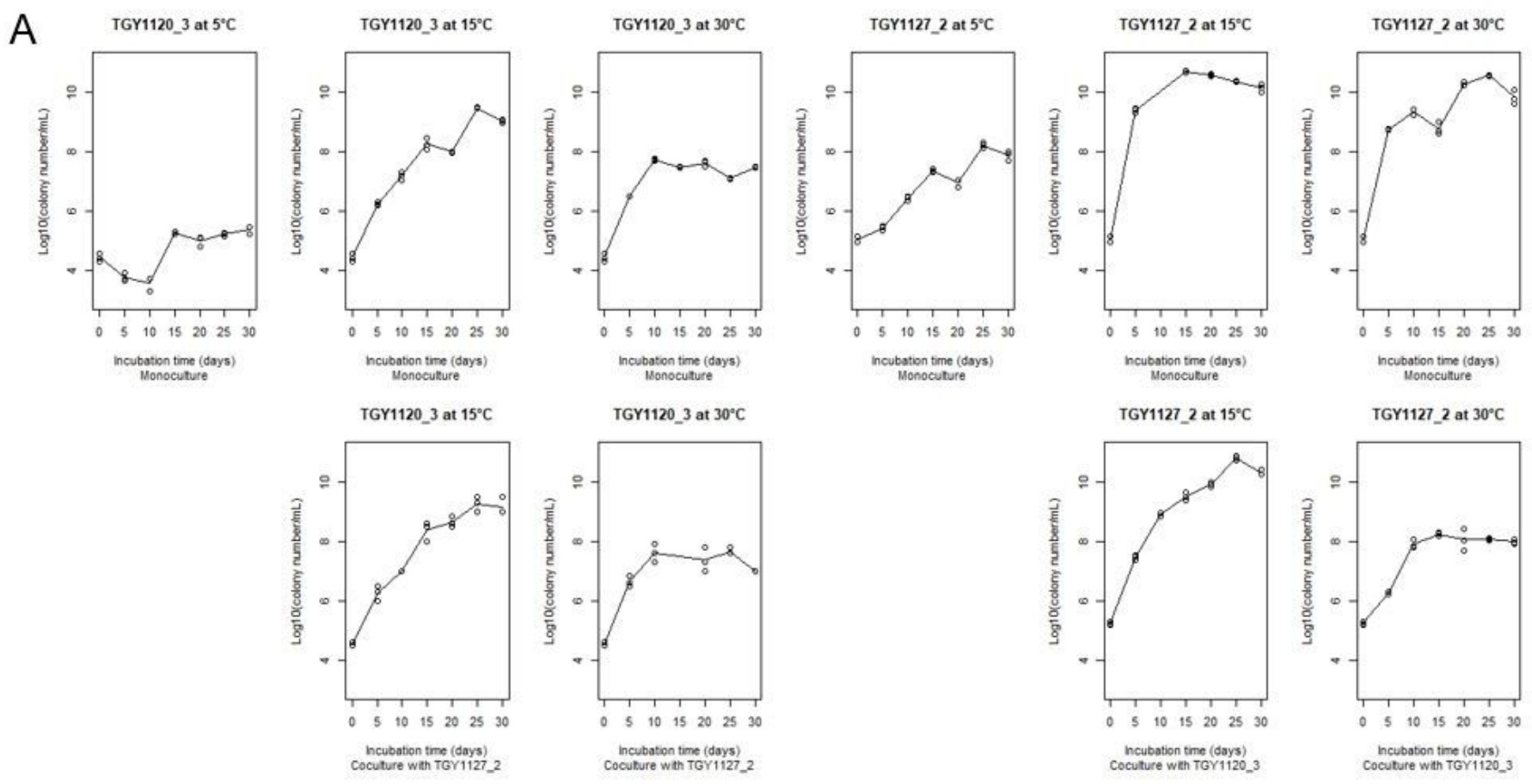

B

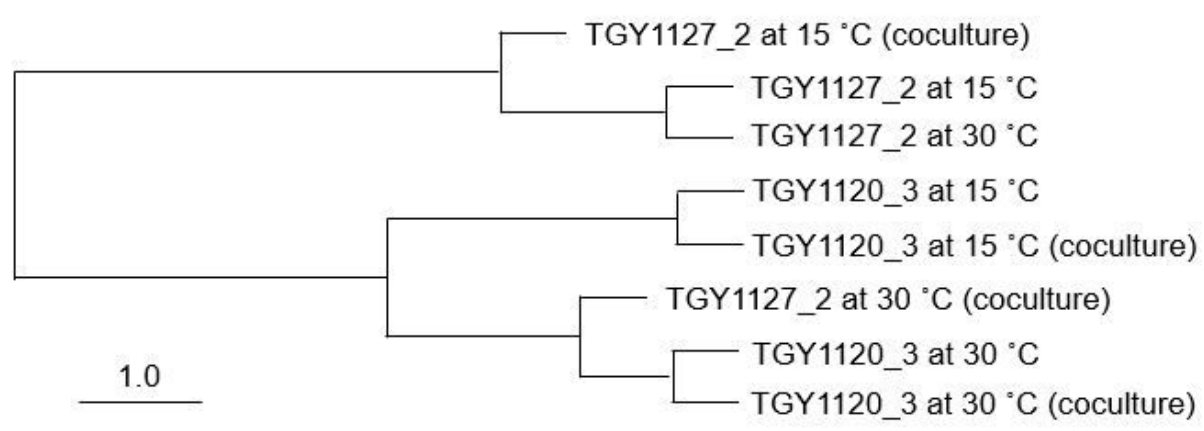

\section{Figure 3}

A) Growth of Kocuria isolates in monoculture and co-cultures in TGY medium. The incubation temperatures were $5{ }^{\circ} \mathrm{C}, 15^{\circ} \mathrm{C}$, and $30^{\circ} \mathrm{C}$. B) Phylogenetic relationships based on the growth patterns of Kocuria isolates under different culture conditions. 

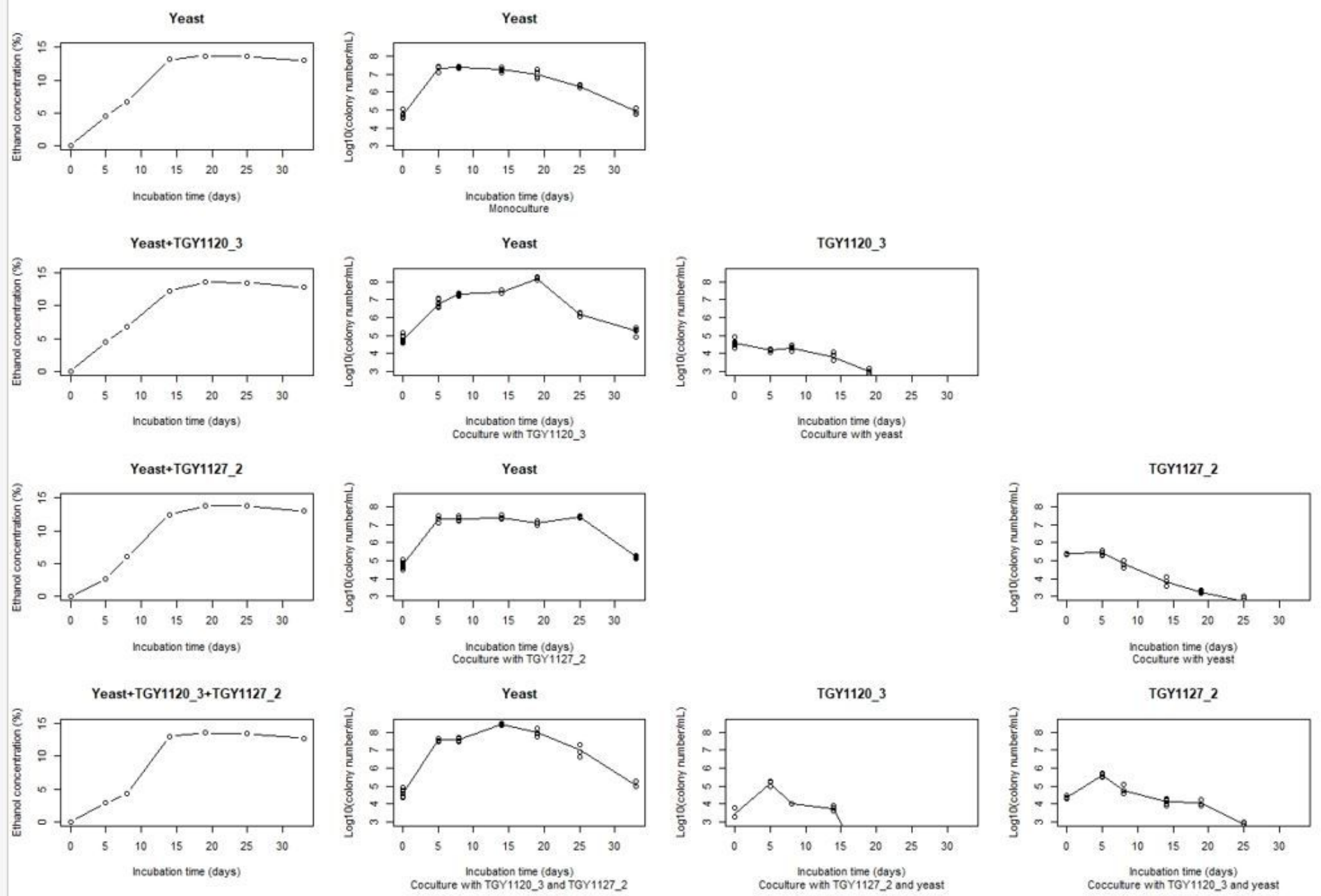

Figure 4

Ethanol concentration and growth of sake yeast, Kocuria isolate TGY1120_3, and TGY1127_2 in monocultures and co-cultures in TGYm2 medium. The incubation temperature was $15^{\circ} \mathrm{C}$. 

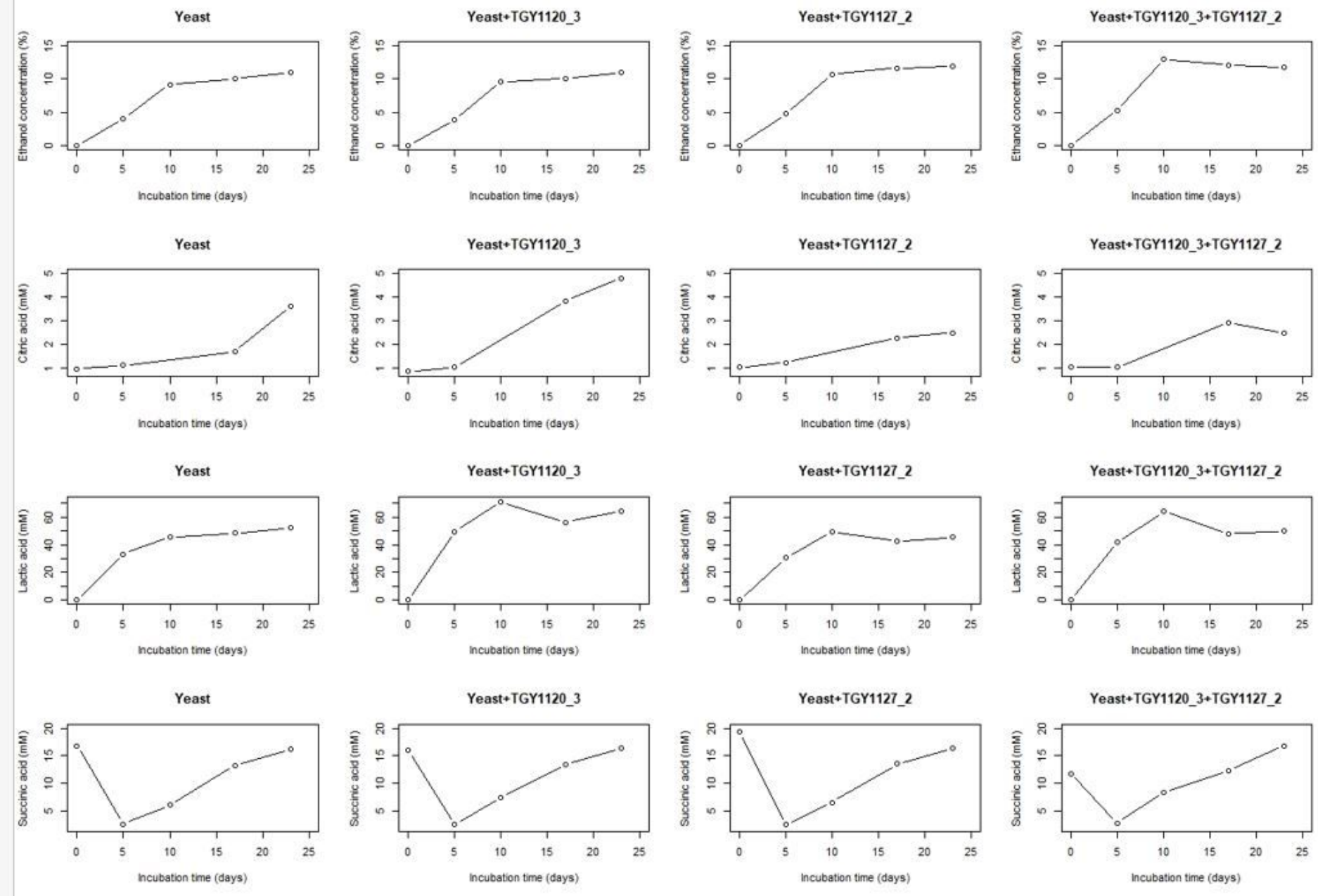

\section{Figure 5}

Changes in ethanol, citric acid, lactic acid, and succinic acid concentrations in monocultures and cocultures in koji solution. The incubation temperature was $15^{\circ} \mathrm{C}$. 


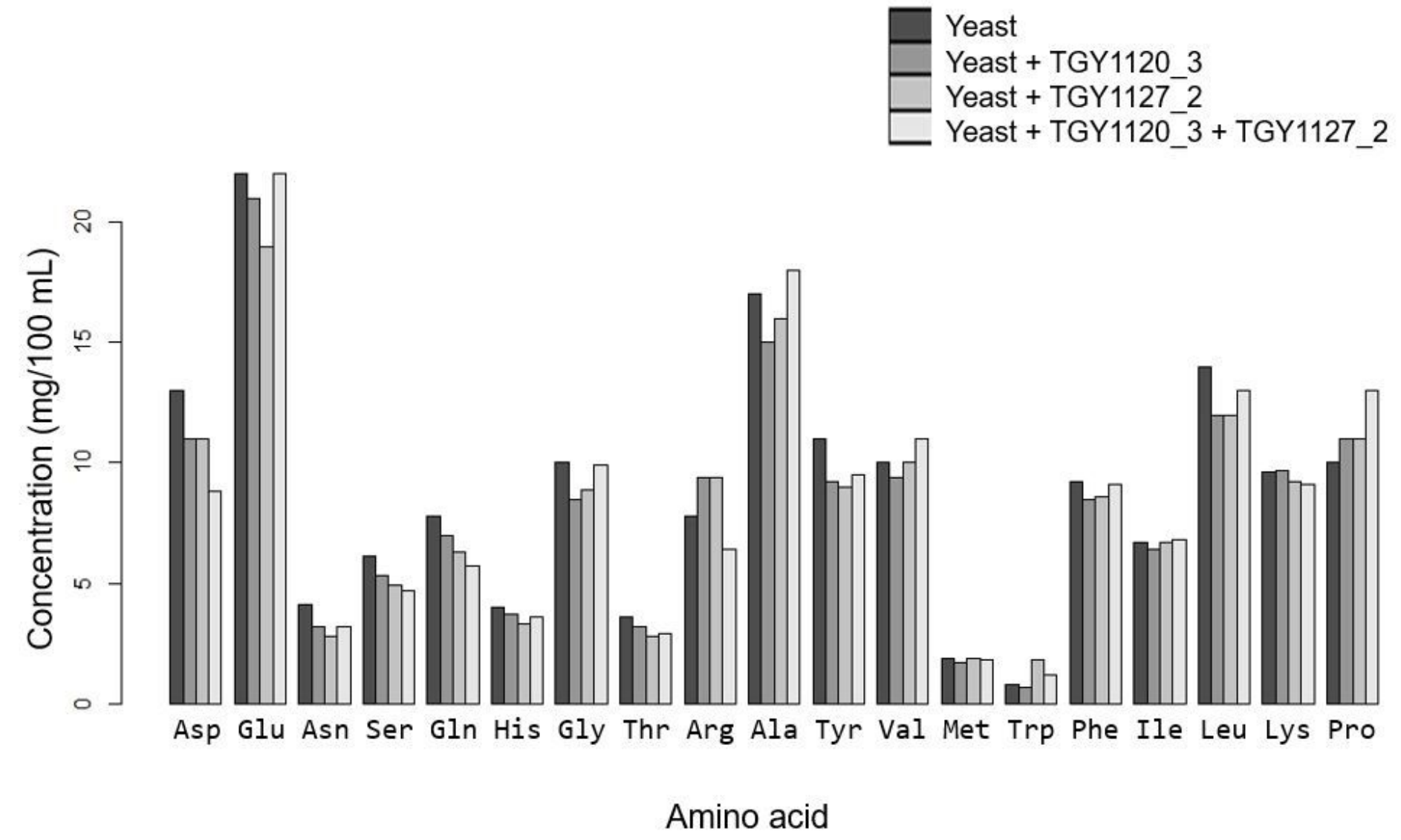

Figure 6

Amino acid compositions of the final cultures of koji solution. 